\title{
EXPERIENCIA EN LA APLICACIÓN DEL PROTOCOLO DE BUENAS PRÁCTICAS EN PARENTALI- DAD POSITIVA DESDE EL EQUIPO TERRITORIAL DE SERVICIOS SOCIALES ÁREA I
}

\author{
Ma Grata Maseda Lodos \\ Consejería de Servicios y Derechos Sociales \\ Equipo de Servicios Sociales Territorial Área I Navia \\ Buenas prácticas en Parentalidad Positiva \\ mariagrata.masedalodos@asturias.org
}

Fecha de Recepción: 27 Febrero 2019

Fecha de Admisión: 30 Abril 2019

\section{RESUMEN}

En el año 2009 se desarrolló en Navia (Asturias) el Programa de Orientación Educativa Familiar (POEF), en fase de pilotaje. En este momento, se comenzó un proceso cíclico de planificación - ejecución - evaluación, como es propio en la práctica de las ciencias sociales. No entendimos este momento como un punto y final, como si fuese la culminación de un proceso, sino que desde su origen tratamos de adaptarnos al contexto, que viene determinado por los cambios sociales y educativos de cada momento, para dar respuestas lo más ajustadas posibles a las necesidades de las personas. Consideramos que en ese momento no se había hecho más que el planteamiento inicial del programa, por lo que continuamos mediante un proceso dialéctico entre la teoría y la práctica, dando forma a una herramienta más adaptada a nuestro entorno de trabajo y a la finalidad última de los servicios sociales que es la compensación de desigualdades, con el objetivo prioritario convertirlo en una medida de acción positiva, entendiendo por acción positiva, aquellas actuaciones 0 intervenciones que se realizan en un colectivo en el que existe una desigualdad con el fin de disminuir o eliminar esta descompensación social.

El trabajo que se presenta podríamos decir que consta de dos partes; en la primera parte, se desarrolla en distintos Ayuntamientos el programa-guía para el desarrollo de competencias emocionales, educativas y parentales desarrollado por la profesora Dra. Raquel-Amaya Martínez González de la Universidad de Oviedo. Desde un primer momento, valoramos el programa como una herramienta necesaria, muy eficaz y valiosa por su carácter multiplicador y preventivo.

En la segunda parte, como respuesta a la convocatoria que presenta el Ministerio de Sanidad, Servicios Sociales e Igualdad y la Federación Española de Municipios y Provincias que tiene por fin promover el ejercicio positivo de la parentalidad y reforzar el apoyo a la parentalidad positiva en las políticas y servicios públicos de los Gobiernos Locales y en el tejido asociativo. La iniciativa era 


\section{EXPERIENCIA EN LA APLICACIÓN DEL PROTOCOLO DE BUENAS PRÁCTICAS EN PARENTALIDAD POSITIVA DESDE EL EQUIPO TERRITORIAL DE SERVICIOS SOCIALES ÁREA I}

una oportunidad clara de optimizar la gestión a favor de la coordinación interdisciplinar, la sensibilización, la formación, la promoción ciudadana y la prevención de situaciones de riesgo; también la invitación a que diferentes agentes sociales sumen a sus objetivos o líneas de actuación esta filosofía; todo ello siguiendo la recomendación del Consejo de Europa 2006.

Palabras clave: competencias emocionales; educativas y parentales; parentalidad positiva; sensibilización; protocolo

\section{ABSTRACT \\ Experience in the application of the protocol of good practices in positive parenting from the territorial team of social services area I in Asturias (Spain). In 2009, the Family Educational Orientation Program (POEF) was developed in Navia (Asturias), during the piloting phase. At this time, a cyclical process of planning - execution - evaluation was started, as it is inherent in social sciences.}

This moment wasn't understand as final point, as if it were the finalization of an adaptation process; However, from its origin, we have tried to adapt to the context. This is determined by the social and educational changes of each moment, to give adapted answers to the needs of people.

At that time, we considered that the only initial approach of the program had been done. This is why we continued through a dialectic process between theory and practice, and we designed an adapted tool to our work environment with the main aim of the social services which is the compensation of inequalities. Furthermore, the priority objective of this tool is become an 'positive action measure', we should understand 'positive action' as those actions or interventions that are carried out in a group in which there is an inequality situation in order to decrease or eliminate this social decompensation.

It could be said that this project has two parts. In the first one, in different municipalities in the area, the 'guide-program for the development of emotional, educational and parental competences' designed by the professor Dr. Raquel-Amaya Martínez González from Oviedo University, is developed by technicians from social services.

From the beginning, we value the program as a necessary tool, very effective and valuable due to its multiplying and preventive impact.

In the second one, after the call by the Ministry of Health, Social Services and Equality and the Spanish Federation of Municipalities and Provinces the 'certificate of positive parenthood' is obtained. Its aims is to promote the positive exercise of parenthood and support positive parenting in the policies and services public of the Local Governments and association networks. The initiative was a clear opportunity to optimize the management in favor of interdisciplinary coordination, awareness, training, citizen promotion and the prevention of risk situations. Besides, different social agents are invited to add this philosophy to their objectives or lines of action. This follows the recommendation of the Council of Europe 2006.

Keywords: emotional; educational and parental competences; positive parenting; sensitization; protocol

\section{INTRODUCCIÓN}

El presente trabajo pretende reflejar los aprendizajes, de carácter tanto teórico como práctico, adquiridos y las experiencias desarrolladas en un contexto determinado durante la última década, en torno a la capacitación parental.

No es un término nuevo ni acabado. No es nuevo en el sentido de que, como bien sabemos, en el 2006, el comité de Ministros de la Comisión Europea lanza una Recomendación reivindicando la importancia de las políticas de apoyo al ejercicio positivo de la parentalidad; refiriéndose al "com- 
portamiento de los padres fundamentado en el interés superior del niño, que cuida, desarrolla sus capacidades, no es violento y ofrece reconocimiento y orientación, incluyendo el establecimiento de límites que permitan el pleno desarrollo del niño, de la niña" , lo que sí es nuevo es el modo en que se concibe el ejercicio de la parentalidad.

$\mathrm{Ni}$ es un concepto acabado, sino que desde su origen trata de adaptarse al contexto que viene determinado por cambios sociales, las necesidades que presenta cada familia, los valores imperantes,... para dar respuestas lo más ajustadas posibles a las necesidades de las personas. De ahí que partamos de entender la orientación familiar como Bisquerrra (2005) entiende la orientación educativa, definiéndola como un:

proceso de ayuda y acompañamiento continuo a todas las personas, en todos sus aspectos, como objeto de potenciar la prevención y el desarrollo humano a lo largo de toda la vida. Esta ayuda se realiza mediante una intervención profesionalizada, basada en principios científicos y filosóficos (2005, p. 2)

De esta definición destacaremos algunos aspectos. Esta orientación a personas con responsabilidades parentales es un proceso continuo en el tiempo, que no proviene únicamente de forma puntual del sistema educativo, del sistema de servicios sociales, ..., sino que también es un agente importante la comunidad, los distintos agentes que de una u otra manera intervienen con las familias. Este proceso de ayuda continuo se dirige a todas las personas con responsabilidades parentales, no sólo a las familias que puedan presentar problemas con sus hijos y/o hijas, y a largo de su vida, ya sea de una manera preventiva o como respuesta a las demandas.

Es importante poner el tema de la parentalidad positiva sobre la mesa y trabajarlo. Hay un cambio conceptual que afecta al núcleo básico de la tarea parental; la necesidad de sustituir autoridad parental por responsabilidad parental. No se pone en cuestión si las figuras parentales deben o no ejercer la autoridad para poder guiar, orientar a nuestros hijos, sino cómo ejercer esa autoridad de modo responsable para preservar los derechos de los más pequeños sin menoscabar los propios, y fomentar la capacidad crítica y de participación de las hijas e hijos, a la vez que se promueve su progresiva autonomía.

\section{CARACTERÍSTICAS DEL SERVICIO}

El Decreto 108/2005 del Mapa Asturiano de Servicios Sociales, regula la ordenación territorial de los servicios sociales en su conjunto, con el principal objetivo de ser un instrumento útil para la planificación y dotación de servicios. La zonificación que marca debe de servir de base a la estructura de la red de atención. Esta ordenación territorial de servicios sociales facilita la cercanía y proximidad de los recursos a las personas usuarias. Así Asturias se divide en 8 áreas, coincidiendo con las áreas sociosanitarias, y el área I, donde se ubica este trabajo, reúne 17 concejos del noroccidente asturiano.

El área I limita al norte con el mar Cantábrico, al sur con Cangas del Narcea, al este con Avilés y al oeste con Galicia. Hay que destacar que es un área con gran extensión, donde los núcleos de población rurales están muy dispersos. Ocupa una extensión de $1.641,5 \mathrm{~km}^{2}$ y tiene una densidad de población de $30 \mathrm{hab} / \mathrm{km}^{2}$, muy por debajo de la media de la región, que se sitúa en los 100 hab./km². A enero de 2018, el no total de habitantes en el área I es de 44933 personas.

Todos los Equipos tienen la misma estructura organizativa. Son equipos interdisciplinares donde hay un/a coordinador/a, 1 pedagogo/a, 1 educador/a, 1 trabajador/a social y un auxiliar administrativo. Con la entrada en vigor de la Ley 39/2006, de 14 de diciembre de Promoción de la autonomía personal y atención a personas en situación de dependencia, pasaron a formar parte de los Equipos el personal valorador de la dependencia. 


\section{EXPERIENCIA EN LA APLICACIÓN DEL PROTOCOLO DE BUENAS PRÁCTICAS EN PARENTALIDAD POSITIVA DESDE EL EQUIPO TERRITORIAL DE SERVICIOS SOCIALES ÁREA I}

Los objetivos de estos grupos interdisciplinares son, entre otras: Promover la igualdad en la distribución de recursos; la supervisión de acuerdos y convenios del Principado con las corporaciones locales para el desarrollo de las prestaciones sociales básicas; apoyar a los SSM en la elaboración de itinerarios individualizados de inserción según la Ley 4 / 2005 de salario social básico; Detectar y analizar la demanda como base para la planificación integrando las informaciones obtenidas en el Área; Proponer, impulsar y ejecutar planes y programas en el ámbito del Área; Participar en el desarrollo de la Ley 39 / 2006 y apoyar y asesorar en el desarrollo de los Planes de Infancia.

\section{APLICACIÓN DEL PROTOCOLO}

Actualmente, la investigación sigue siendo una de las asignaturas pendientes en los servicios sociales, pues si bien es cierto que cada vez está más presente, aún no ha calado, a mi modo de ver, como sería lo adecuado en un ámbito como el nuestro.

Resulta indiscutible que cada vez más profesionales de los servicios sociales participan en estudios de investigación, pero también hemos de reconocer que todavía la investigación no tiene el protagonismo que debería en nuestro ámbito, quizás porque es necesario que se produzca un cambio de actitud dentro del colectivo. Si bien aceptamos, demandamos, formación rigurosa, nos mostramos muy reticentes a repensar nuestra práctica y a evaluar las intervenciones.

Son muchas las ventajas de los protocolos basados en la evidencia. Lo que pudimos observar durante este escaso año y medio es que facilitan la comunicación intercentros, intersectores, y con ello no solo se posibilita el mejor conocimiento de profesionales, sino que también sirve para mejorar el posicionamiento de los distintos servicios de cara a la ciudadanía y a los responsables políticos que en último término son los que tienen que apostar por políticas sociales.

El protocolo de buenas prácticas en parentalidad positiva, nos permitió pararnos a pensar en el trabajo profesional que se estaba desarrollando, directa o indirectamente con las familias, y el propio servicio. Como se comentó anteriormente, desde el área I llevamos 10 años trabajando la orientación educativa familiar, y durante estos 10 años se añalizó en numerosas ocasiones la herramienta de trabajo, el "programa guía para el desarrollo en competencias emocionales, educativas y parentales", de la profesora Raquel Amaya Martínez, pero no se hizo lo mismo con nuestra propia práctica ni con el servicio en el que nos situamos.

La protocolización, entendida como una metodología o como una técnica, precisa un aprendizaje y un entrenamiento práctico como cualquier otro aspecto, y precisa de tiempo. En el ámbito en el que trabajamos, están instaladas rutinas de trabajo orientadas más al resolver que al planificar, y los tiempos son muy escasos en relación a la demanda existente. Estamos todavía al inicio del camino pero ya tenemos el "porqué" de nuestro hacer, y la evidencia de que este hacer tiene que ser de manera coordinada entre todos los servicios y de los distintos ámbitos.

\section{RESULTADOS: INFORME FINAL}

Son muchos años trabajando con las familias, pero pasó todavía poco tiempo desde que los primeros Ayuntamientos del área I: Coaña, Boal, Pesoz, Illano, Grandas de Salime, o las primeras entidades como la Fundación EDES, aplicaron el protocolo, para poder hablar de resultados. Lo que sí podemos trasladar es de lo que esta línea de trabajo nos ofreció a aquellas personas y/o servicios que nos sumamos a ella.

\section{Una visión integral del trabajo.}

El participar en la solicitud de este reconocimiento permite al Equipo Impulsor abordar un tema, como es el de la Capacitación Parental, desde una óptica transversal e integradora, por ser un tema que no se ubica en servicios sociales, o en salud únicamente, sino que cualquier servicio municipal 
que trabaja con personas está actuando sobre un miembro de la unidad familiar. Por otra parte, y no menos importante, es necesario en territorios tan pequeños como en los que nos ubicamos, que entendamos que la dinamización del tejido social sólo será posible si todos nos situamos en el mismo plano y en una misma línea, cada uno desde el ámbito o parcela que le corresponda. Esta idea de partida fue el motivo de escoger, en la mayor parte de los Planes de Mejora presentados, el objetivo de "Visibilizar la importancia y necesidad de trabajar con las familias desde la perspectiva de la parentalidad positiva", para poner en valor la importancia que todos los agentes sociales y la población general se impliquen en esta línea de trabajo.

\section{Optimizar proyectos ya existentes en el municipio bajo la óptica de la parentalidad positiva.}

El hacer el análisis de nuestro servicio, de nuestro trabajo, nos facilitó ver la vinculación entre distintos programas, destacando aquí, la vinculación entre las ciudades amigas de la infancia y la parentalidad positiva. Si bien es algo intrínseco a los propios Planes de Infancia de los distintos Aytos, se materializó en el Plan de Infancia 2016-2020 del municipio de Coaña, donde dentro del plan se trabaja además de la sensibilización en derechos, distintas intervenciones de prevención e intervención, que incluyen el trabajo con familias, centros educativos y la formación del personal del propio Ayuntamiento.

Una ciudad amiga de la infancia es un pueblo, comunidad o gobierno local comprometido con el cumplimiento de los derechos de las y los niños, las y los adolescentes, tal y como se establece en la Convención sobre los Derechos del Niño. En nuestra área hay 5 Ayuntamientos que bien están reconocidos o están en proceso de reconocimiento como "Ciudades Amigas de la Infancia".

El conocimiento científico actual nos permite concluir que la mejor forma de garantizar social y jurídicamente la protección a la infancia es promover su autonomía como sujetos. De esta manera podrán ir construyendo progresivamente una percepción de control acerca de su situación personal y de su proyección. Así, los programas de prevención a través de una orientación educativa a padres y madres dirigidos a capacitarles en el adecuado ejercicio de una parentalidad positiva han demostrado una gran eficacia y dan respuesta a una demanda formulada por las propias familias; y el conocimiento práctico, nos permite concluir que ésta es la línea de trabajo, por lo que ahora nos corresponde coordinarnos con otros Planes de Infancia del Área y trasladarles lo que fuimos construyendo y aprendiendo.

\section{El trabajo en red.}

Entendemos el trabajo en red como aquellas relaciones dialécticas que se producen entre un conjunto de agentes sociales que confluyen en un territorio y que ante un objetivo común (bienestar y calidad de vida) deciden participar en un proceso de mejora que redundará en un mejor conocimiento de la realidad, en intervenciones más eficaces y una evaluación óptima de los resultados esperados así como más satisfacción en el desarrollo profesional y sobre todo, en una mayor cohesión territorial y sentimiento de pertenencia a una comunidad de todas las personas y agentes participantes. Situados en esta metodología de trabajo, desde las Escuelas Municipales de salud en coordinación con el Área Sanitaria I consiguieron, que durante una semana, del 5 al 11 de novimbre de 2018, la parentalidad positiva fuese la propuesta fundamental, convirtiéndola en el tema de la V Semana Saludable en el Noroccidente, bajo el lema, "Familiarmente hablando".

\section{PLAN DE MEJORA}

La situación de partida de los distintos Planes de Mejora son unos servicios y programas autonómicos y municipales que ejercen una gestión socioeducativa importante en el territorio donde juega un papel destacado la existencia de un trabajo previo de 10 años en capacitación parental y 


\section{EXPERIENCIA EN LA APLICACIÓN DEL PROTOCOLO DE BUENAS PRÁCTICAS EN PARENTALIDAD POSITIVA DESDE EL EQUIPO TERRITORIAL DE SERVICIOS SOCIALES ÁREA I}

varios Planes Integrales de Infancia y Adolescencia que han sido reconocidos por UNICEF España con el Sello Ciudad Amiga de la Infancia.

Los Servicios Sociales Autonómicos se han dirigido a los servicios municipales y a Entidades no lucrativas del territorio, con el fin de aprovechar este potencial a favor de la parentalidad positiva y teniendo en cuenta las herramientas que brinda el Ministerio de Sanidad, Servicios Sociales e Igualdad y la FEMP que permiten articular de forma eficaz cada esfuerzo o línea de trabajo en esta materia.

Por tanto, la iniciativa es una oportunidad clara de optimizar la gestión a favor de la coordinación interdisciplinar, la sensibilización, la formación, la promoción ciudadana y la prevención de situaciones de riesgo. También la invitación a que diferentes agentes sociales sumen a sus objetivos o líneas de actuación esta filosofía; todo ello siguiendo la recomendación del Consejo de Europa 2006.

\section{CONCLUSIONES}

A través de la solicitud del Reconomiento a la Promoción de la Parentalidad Positiva se nos dibujó un marco inmejorable para intentar avanzar en la línea de la parentalidad positiva y de visibilizar el trabajo que llevamos años desarrollando. Esto a su vez conlleva una serie de exigencias, como es la necesidad del trabajo conjunto entre administraciones, la local y la autonómica. Una coordinación entre agentes, todas y todos intervenimos en el mismo colectivo: las familias, y por lo tanto es un tema que a todos los profesionales nos incumbe. Aquellas entidades que trabajen en el sello deberían ser entidades, concejos sensibilizados, donde repiensen los servicios que ofrecen y los hagan familiarmente accesibles.

\section{REFERENCIAS BIBLIOGRÁFICAS}

BISQUERRA, R. (2005). La educación emocional en la formación del profesorado.

Colectivo loé (2003) "Investigación - acción participativa: propuesta para un ejercicio activo de la ciudadanía"

CONSEJO DE EUROPA (2006). Recomendación Rec (2006) 19 del Comité de Ministros a los Estados Miembros sobre políticas de apoyo a la Parentalidad Positiva

MARTA LLOBET • FERRÁN CORTÉS • ROSA Mํㅡㄹ ALEMANY PORTULARIA 4, 2004, [445-454], ISSN 1578-0236. ( ) UNIVERSIDAD DE HUELVA

MARTíNEZ GONZÁLEZ, R.A., Pérez Herrero, H. y Álvarez Blanco, L. (2007). Estrategias para prevenir $y$ afrontar conflictos en las relaciones familiares (padres e hijos). Madrid: Ministerio de Trabajo y Asuntos Sociales. http://www.observatoriodelainfancia.msssi.gob.es/productos/pdf/

MARTíNEZ GONZÁLEZ, R.A. (2009). Programa-Guía para el Desarrollo de Competencias

Emocionales, Educativas y Parentales. Madrid: Ministerio de Sanidad y Política

Social.

MARTÍNEZ GONZÁLEZ, R.A. y BECEDÓNIZ VÁZQUEZ, C. M. (2009). Orientación Educativa

para la Vida Familiar como Medida de Apoyo para el Desempeño de la Parentalidad

Positiva. Intervención Psicosocial, 18 (2) 97-112.

MARTínEZ GONZÁLEZ, R.A. (Coord.) (2010). Parentalidad Positiva en Asturias. Oviedo: Consejería de Bienestar Social y Vivienda del Principado de Asturias y Ministerio de Sanidad, Política Social e Igualdad. http://www.observatoriodelainfanciadeasturias.es/biblioteca?pag=6

VILLASANTE, T. R. (2006) "Desbordes creativos. Estilos y estrategias para la transformación social". Catarata. Madrid.

http://familiasenpositivo.org/

www.fantova.net 